Research Paper

\title{
Airway Administration of Vascular Endothelial Growth Factor siRNAs Induces Transient Airspace Enlargement in Mice
}

\author{
Yusuke Takahashi ${ }^{1}$, Yotaro Izumi ${ }^{\bowtie}{ }^{\bowtie}$, Mitsutomo Kohno $^{1}$, Eiji Ikeda ${ }^{2}$, Hiroaki Nomori ${ }^{1}$ \\ 1. Division of General Thoracic Surgery, Department of Surgery, School of Medicine, Keio University, Tokyo, Japan. \\ 2. Department of Pathology, Yamaguchi University Graduate School of Medicine, Yamaguchi, Japan.
}

$\triangle$ Corresponding author: Yotaro Izumi, Division of General Thoracic Surgery, Department of Surgery, School of Medicine, Keio University, 35 Shinanomachi, Shinjuku-ku, Tokyo 160-8582, Japan. TEL: +81-5363-3806, FAX: +81-5363-3499. E-mail: yotaro2008@gmail.com.

(c) Ivyspring International Publisher. This is an open-access article distributed under the terms of the Creative Commons License (http://creativecommons.org/ licenses/by-nc-nd/3.0/). Reproduction is permitted for personal, noncommercial use, provided that the article is in whole, unmodified, and properly cited.

Received: 2013.07.08; Accepted: 2013.08.26; Published: 2013.09.27

\begin{abstract}
Purpose: Reduction in the level of vascular endothelial growth factor (VEGF) has been implicated in the pathogenesis of pulmonary emphysema. To this end, pharmacological VEGF receptor blockade, and the Cre-lox system models have been utilized to study the effects of VEGF depletion in the lung. These models generally reproduce air space enlargement resembling clinical emphysema. Here we report a potentially more readily available model of lung targeted VEGF depletion by airway administration of VEGF small inhibitory RNA oligonucleotides (siRNAs) in mice.

Methods: Airway administration of VEGF siRNAs were done in C57BL/6 mice. The lungs were removed for histology and protein analysis 2 , and 4 days later. Airspace enlargement was evaluated by lung volume measurement, and histological analyses. VEGF levels were analyzed by western blot and immunohistochemistry.

Results: Airway administration of VEGF siRNAs induced transient air space enlargement in the mouse lung morphologically resembling the previously reported models of pulmonary emphysema. VEGF expression was significantly reduced in the lung, particularly in the alveolar septal cells. We also found that in this particular model, sequential airway administration of recombinant VEGF protein attenuated this air space enlargement. Additionally, we found that airway administration of $\mathrm{DCl}$, a combination of dexamethasone, 3'-5'-cyclic adenosine monophosphate, and isobutylmethylxanthine attenuated the air space enlargement in this particular model, at least in part through the recovery of lung VEGF expression.

Conclusions: The pathogenesis of pulmonary emphysema is likely to be multifaceted, but the present mouse model may be useful in dissecting the involvement of VEGF in pulmonary emphysema.
\end{abstract}

Key words: vascular endothelial growth factor, emphysema, mouse model.

\section{Introduction}

Pulmonary emphysema, one of the major components of chronic obstructive pulmonary disease, is a syndrome which presumably results from multifactorial causes [1]. Morphologically, destruction of alveolar structure including the microvasculature is associated with airspace enlargement and subsequent loss of gas exchange area. Although cigarette smoke is considered to be one of the main etiologic factors of emphysema, the pathogenesis remains unclear. Several mechanism are thought to be involved in the development of emphysema, including airway inflammation, protease/antiprotease imbalance, and vascu- 
lature impairment.

Among various organs, the lung contains the highest level of vascular endothelial growth factor (VEGF) transcripts [2]. The progression of alveolar septal destruction in emphysema may be due to a facilitative loop of apoptosis caused by reduction in growth factors such as VEGF, accompanied by oxidative stress and matrix proteolysis which induces inflammatory responses and further decreases growth factor availability [3]. Indeed, the level of VEGF is known to be decreased in the lungs of emphysema patients $[4,5]$. These findings suggest that downregulation of VEGF in the lung may at least in part be involved in the pathogenesis of emphysema, presumably by reducing the ability of pulmonary epithelial and endothelial cells to recover from injuries inflicted by external stimuli such as oxidants or cigarette smoke.

Pharmacological VEGF receptor blockade models have been utilized to study the effects of VEGF depletion in the lung [6-9]. Lung specific VEGF depletion has been reported using the Cre-lox system [10]. These models generally reproduce air space enlargement resembling clinical emphysema. Here we report a potentially more readily available model of lung targeted VEGF depletion by airway administration of VEGF small inhibitory RNA oligonucleotides (siRNAs) which induced transient air space enlargement in the mouse lung. We also show that in this particular model, sequential airway administration of recombinant VEGF protein attenuated this air space enlargement. In addition, since previous studies showed that DCI, a combination of dexamethasone, 3'-5'-cyclic adenosine monophosphate, and isobutylmethylxanthine, facilitated differentiation of type II alveolar cells in vitro [11], we also evaluated the effects of DCI on air space enlargement in this particular model.

\section{Matrials and methods}

\section{Animals}

Specific pathogen-free, 9-week-old, inbred, male C57BL/ 6 mice, weighing approximately $20 \mathrm{~g}$, were purchased from CLEA Japan, Inc. (Tokyo, Japan). The mice were kept in a 12-h light/12-h dark cycle with free access to food and water. The mice were randomly assigned to five experimental groups, airway administration of VEGF siRNAs (siVEGF\#2, and siVEGF\#3 groups), airway administration of thyroid transcription factor-1 (TTF-1) siRNAs (siTTF-1\#2, and siTTF-1\#4 groups), airway administration of VEGF siRNAs followed 2 days later by airway administration of recombinant mouse VEGF 164 (+rec VEGF group), airway administration of VEGF siRNAs fol- lowed 2 days later by airway administration of DCI, a mixture of dexamethasone $(10 \mathrm{nM})$, 8-bromo-3'-5'cyclic adenosine monophosphate (cAMP) $(0.1 \mathrm{mM})$, and isobutylmethylxanthine (0.1 mM) (+DCI group), and the control group (CON group), in which measurements were taken 4 days after airway administration of a nonsilencing oligonucleotide with Infasurf.

The mice were weighed, and were observed daily for any signs of distress or changes in behaviour. The mice were sacrificed at respective time points by injection of $10 \mathrm{mg} / \mathrm{kg}$ of xylazine and $100 \mathrm{mg} / \mathrm{kg}$ of ketamine, followed by exanguination from the inferior vena cava.

All experimental protocols were approved by the Committee on the Ethics of Animal Experiments at School of Medicine, Keio University (protocol No. 09141), and were carried out in accordance with Guidelines for Animal Experiments issued by the School of Medicine, Keio University Experimental Animal Center.

\section{Selection of silencing small inhibitory RNA oligonucleotides}

Three VEGF silencing small inhibitory RNA oligonucleotides (siRNAs), si\#VEGF1 through si\#3, were synthesized and purified by Invitrogen (Carlsbad, CA). Briefly, a single strand of RNA was synthesized by Phosphoramidite method. After synthesis of single strand RNA, RNA was deprotected in two steps from base and phosphate protecting group, and 2 '-hydroxyl function protecting. Single strand RNA was desalted or purified after deprotection. The single strand RNA was annealed as siRNA.

A mouse lung carcinoma cell line, Lewis lung carcinoma cell line (LLC) (Dainippon Sumitomo Pharma Co., Tokyo, Japan), was used to evaluate the efficacy of VEGF silencing oligoneucleotides. The cells were maintained in Dulbecco's modified Eagle's medium (DMEM) supplemented with 5\% fetal bovine serum, under $5 \% \mathrm{CO}^{2}$. By western analysis using LLC, densitometry of VEGF standardized to bet-actin, represented as mean \pm standard deviation, were $1.3 \pm 0.5$ in non-silence, $1.1 \pm 0.4$ in siVEGF\#1, $0.6 \pm 0.3$ in siVEGF\#2, and $0.5 \pm 0.3$ in siVEGF\#3, respectively $(n=4)$. Thus, oligonucleotides siVEGF\#2, and siVEGF\#3 were found to be significantly effective. Sequences of siVEGF\#2 and siVEGF\#3 were (5'-UAGGAAGCUCAUCUCUCCUAUGUGC) and (5'-AAGUACGUUCGUUUAACUCAAGCUG), respectively. TTF-1 siRNAs, siTTF-1\#2 and siTTF-1\#4 (Invitrogen, Carlsbad, CA) were selected as previously described [12]. These siRNAs have been shown to effectively reduce TTF-1 expression level in the lung. Sequences of siTTF-1\#2 and siTTF-1\#4 were (5'-UUGAAACGUCGCUCGAGCUCGUACA-3') and 
(5'-GCUACAAGAUGAAGCGCCGGCUAA-3'), respectively. As a control nonsilencing oligonucleotide, stealth RNAi negative control duplex (Invitrogen) was used.

\section{Airway administration of materials}

The mice were lightly anesthetized with $3 \mathrm{mg} / \mathrm{kg}$ of xylazine and $30 \mathrm{mg} / \mathrm{kg}$ of ketamine. Each material was administered into the nasal orifices as previously reported [12] using a microliter pipetter. The materials were mixed with Infasurf (Forest Pharmaceuticals, St. Louis, MO), a natural surfactant product obtained from calf lung lavage in buffered saline, as a total of approximately 20 microliters. Each administration was finished within 20 minutes.

For transient VEGF or TTF-1 silencing, $35 \mathrm{mg}$ per kilogram of the respective siRNAs were administered. The dosage was extrapolated from the previously reported in vitro data [11], in vivo data [13, 14], and from our own experiences in the comparisons of in vitro versus in vivo gene silencing using siRNAs. The CON group was administered $35 \mathrm{mg}$ per kilogram of nonsilencing oligonucleotide with Infasurf.

The +recVEGF group was administered VEGF siRNA as described above followed 2 days later by administration of $0.05 \mathrm{mg} / \mathrm{kg}$ of recombinant murine VEGF 164 [15] mixed with Infasurf as a total of 10 microliters. The +DCI group was administered VEGF siRNAs as described above followed 2 days later by DCI, a mixture of dexamethasone (500 nanogram/gram, Sigma), 8-bromo-cAMP (5 microgram/gram, Santa Cruz), and 3-isobutyl-1-methylxanthine (12.5microgram/gram, Calbiochem), mixed with Infasurf as a total of $10 \mathrm{mi}-$ croliters.

\section{Histological analyses}

For histological analyses, the right lungs were inflated with intratracheal instillation of $10 \%$ buffered formalin at a constant pressure of $20 \mathrm{cmH}_{2} \mathrm{O}$, allowing for homogeneous expansion of lung parenchyma. The bronchus was tied under pressure, and the lung was fixed for 48 hours. Total lung volume was measured from the fixed specimen by volume displacement as described by Scherle [16], and was normalized to the body weight as lung volume index (LVI). The paraffin-embedded tissue were sectioned and prepared for histological analysis. After fixation, 5 micrometer sections were made and stained with hematoxylin and eosin. The mean linear intercept, as a measure of interalveolar wall distance, was determined by light microscopy at a total magnification of $x 100$. The mean linear intercept was obtained by the average number of intercepts encountered in seventy-two lines drawn across the lung section per each mouse, as described
$[7,17]$. Morphologically, alveoli were identified as polyhedral, cup, or wedged-shaped terminal air spaces with discrete septae [18]. Five fields were analyzed per animal in four animals. For each analysis, one section was randomly selected per animal, and five 100 fold magnification fields were randomly selected per section. The slides were coded and masked for identify, and were examined by YT and YI.

\section{Western blot analysis for VEGF and TTF-I}

For western blot analyses, the left lungs were resected at respective time points, blotted dry, immediately snap frozen in liquid nitrogen, and stored at -80 degrees Celsius. Western blot analysis for VEGF protein was performed according to a standard protocol. Briefly, lung tissue was lysed with a denatureing RIPA buffer (Sigma, Stockholm, Swaden) and the lysate was centrifuged at $14,000 \mathrm{rpm}$ for 15 minutes at 4 degrees Celsius and the supernatant was mixed with Laemmli buffer and applied to SDS-PAGE gels. The proteins were separated by 12.5\% SDS-PAGE under reducing conditions and then transfered to PVDF membrane for 90 minutes at $90 \mathrm{~V}$ using HorizBlot system (ATTO, Tokyo, Japan). After blocking non-specific reactions with Block Ace (Dainippon Pharmaceutical, Osaka, Japan), the primary antibody for VEGF ( sc507: Santa Cruz biotechnology, Santa Cruz, CA), TTF-1 (H-190: Santa Cruz biotechnology) or antibody for beta actin (Abcam, Cambridge, UK) was incubated with the blots for 1 hour at room temperature. Bands were detected by enhanced chemiluminescence using ECL Western blotting detection reagents (GE Healthcare, Buckinghamshire, UK). Bands were quantified using Image J $(\mathrm{NIH}$, Bethesda, MD). Values were normalized to beta actin.

\section{Immunohistochemistry and terminal deox- ynucleotidyl transferase-mediated nick end labeling (TUNEL) staining}

Immunohistochemistry for VEGF, and Ki-67 was performed as follows. The primary antibodies used were: anti-VEGF rabbit polyclonal antibody (10 microgram/mL: ABS82, Millipore, Billerica, MA) and anti-Ki-67 rabbit polyclonal antibody (10 microgram/mL: ab15580, Abcam, Cambridge, MA). Secondary antibodies used were: anti-rabbit Ig Immpress (Vector Laboratories, Burlingame, CA, USA) for both. Then they were visualized with DAB (Sigma). Staining positive nuclei were counted and expressed in proportion to the number of nuclei in alveolar septal cells. The TUNEL staining was done using deoxyneucleotidyl transferase (TdT) and biotinylated 2'-deoxyuridine 5'-triphosphate ([dUTP] Roche Applied Science, Indianapolis, IN). Counter 
staining was done with Mayer's hematoxylin. For each analysis, one section was randomly selected per animal, and 5, 200 fold magnification fields were randomly selected per section. The slides were coded and masked for identity, and were examined by YT, and EI.

\section{Statistical analysis}

Data are expressed as means \pm standard deviations. Since the animals were sacrificed for respective time point measurements, Mann-Whiteny U-test was used to assess differences between each group and/or time point followed by the post hoc Sidak correction. $P$ values less than 0.05 were considered to be statistically significant. The statistical analysis was performed using StatView software (StatView5.0; Abacus, Berkeley, CA).

\section{Results}

\section{Histological analyses}

Histological analyses showed that airway ad- ministration of VEGF siRNAs induced enlargement of the alveolar air spaces resembling emphysematous changes. These changes were apparent on days 2 and 4 after administration, and tended to recover at day 7 . The magnitude of air space enlargement was similar between the two siRNA sequences, siVEGF\#2 and sVEGFi\#3 (Fig. 1 A, B). Airway administration of recombinant VEGF protein in Infasurf 2 days after administration of VEGF siRNAs attenuated the airspace enlargement on day 4 (Fig. 1 C). Airway administration of DCI in Infasurf 2 days after administration of VEGF siRNAs also attenuated the airspace enlargement on day 4, similar to recombinant VEGF protein administration (Fig. 1 C). Administration of recombinant VEGF or DCI 2 days after a nonsilencing oligonucleotide administration did not induce any apparent changes on histology at day 4 (data not shown).
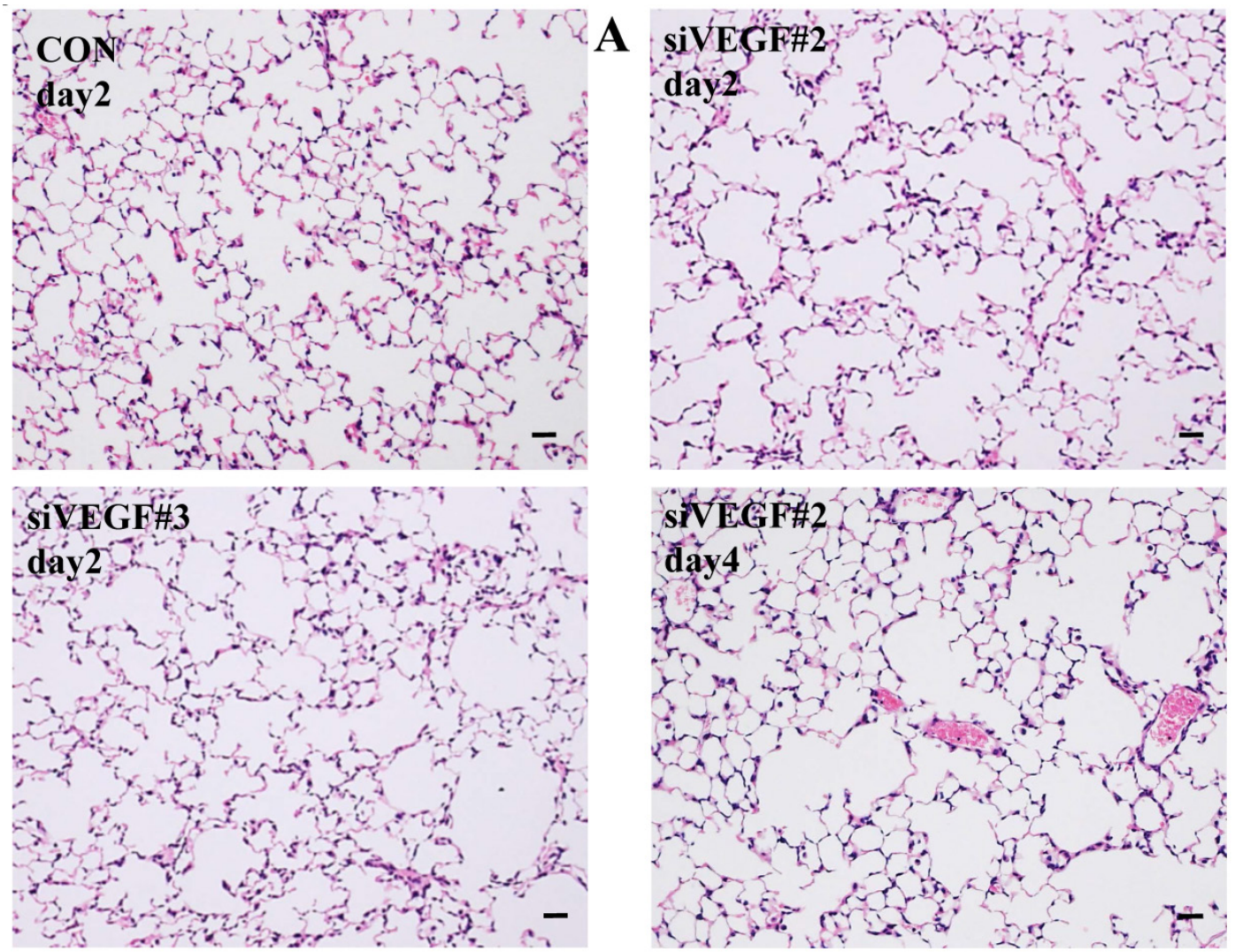

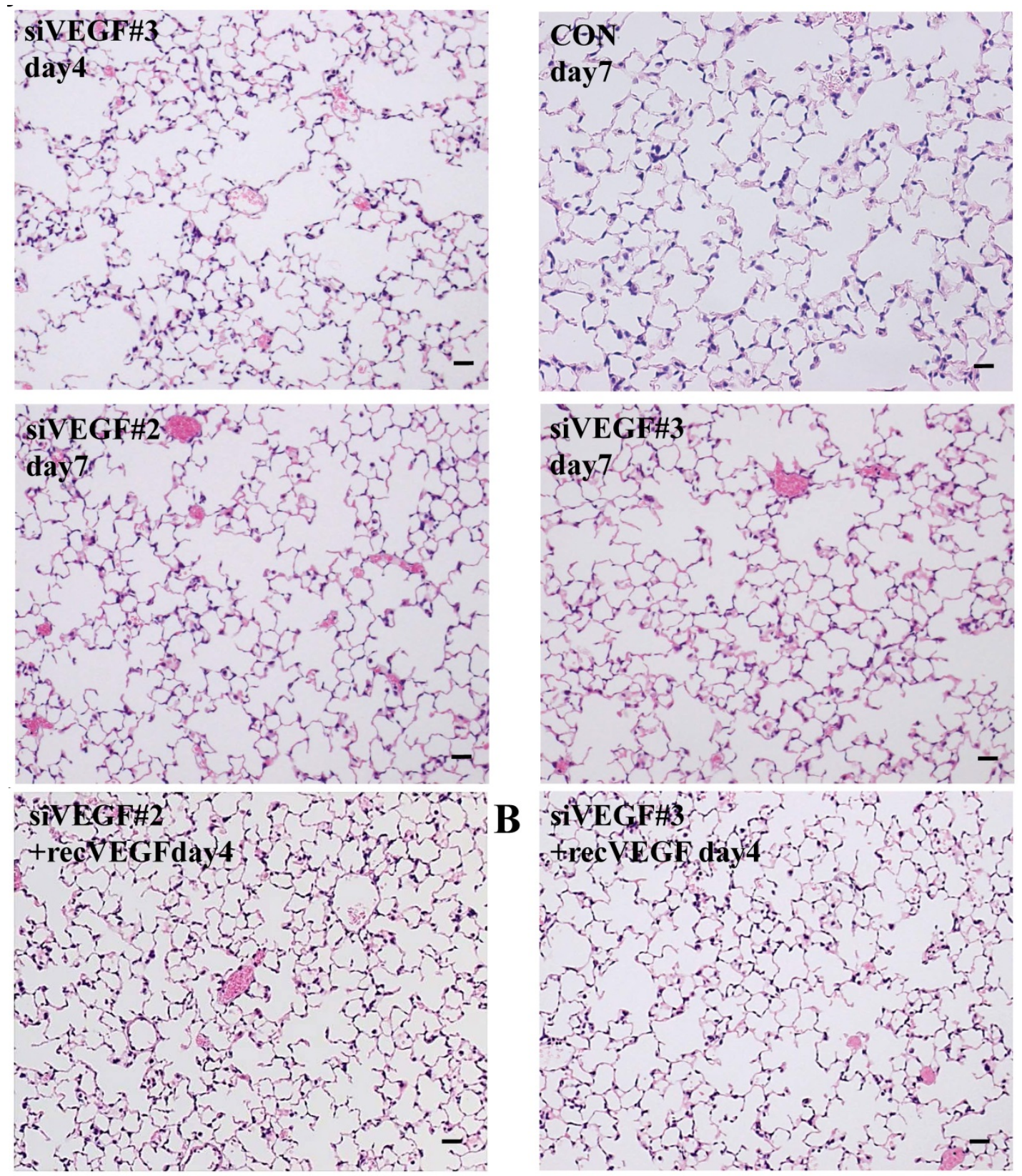

\section{B}
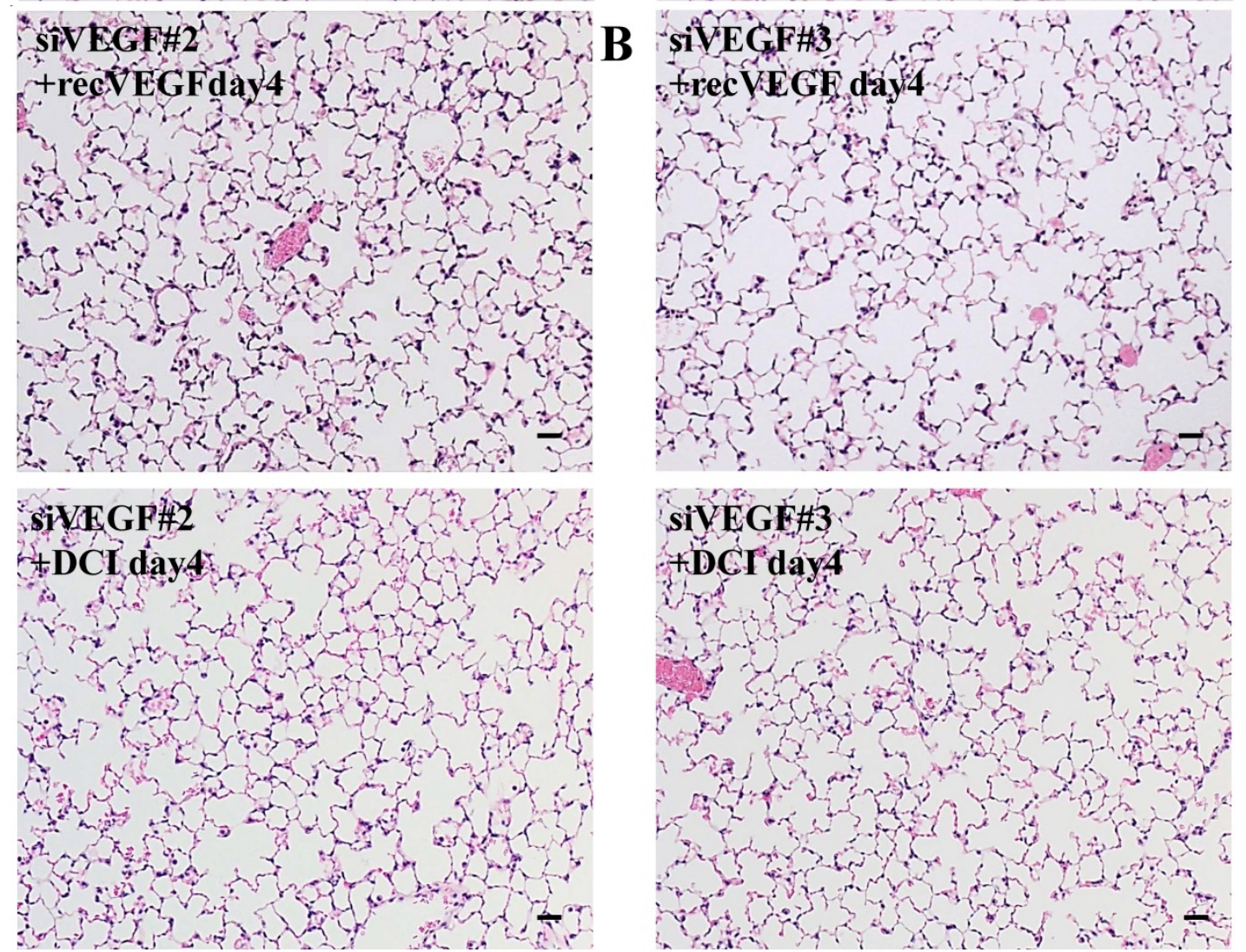

Fig I. Morphological changes after administration of vascular endothelial growth factor small inhibitory RNAs (VEGF siRNAs). (A) Airway administration of 2 sequences of VEGF siRNAs, siVEGF\#2 and \#4 in surface active material Infasurf, induced enlargement of the alveolar air spaces resembling emphysematous changes in comparison to the group administered a nonsilencing oligoneucleotide (CON). These changes were apparent on days 2 and 4 after administration, and tended to recover at day 7. (B) Airway administration of recombinant VEGF, or DCl, a mixture of dexamethasone (IOnM), 8-bromo-3'-5'-cyclic adenosine monophosphate (cAMP) $(0.1 \mathrm{mM})$, and isobutylmethylxanthine $(0.1 \mathrm{mM})$, in Infasurf on day 2 after administration of VEGF siRNAs attenuated the airspace enlargement on days 4 and 7. Haematoxylin and eosin staining, scale bars 100 microns. 
Air space enlargement on histology was quantified by mean linear intercept. Mean linear intercept was significantly decreased in comparison to control at days 2 and 4 after VEGF siRNAs administration. There was a tendency towards recovery at day 7 . Mean linear intercept at day 4 was significantly increased and approached the control level in the groups which received subsequent recombinant VEGF or DCI on day 2, in comparison to the groups which received only VEGF siRNAs (Fig. 2 A).

The number of alveoli per field was significantly decreased in comparison to control at days 2 and 4 after VEGF siRNAs administration. There was a tendency towards recovery at day 7 . Administration of recombinant VEGF or DCI 2 days after VEGF siRNA administration significantly increased the number of alveoli per field at day 4 in comparison to the groups which received only VEGF siRNAs, similar to the level of control (Fig. 2 B).

In agreement with the air space enlargement seen in the histological analyses, LVI was also significantly increased in comparison to control at days 2 and 4 after VEGF siRNAs administration. There was a tendency towards recovery at day 7 . Sequential administration of recombinant VEGF or DCI 2 days after VEGF siRNA administration significantly attenuated the increase in LVI at day 4 and maintained it to a level similar to the control group (Fig. 2 C).
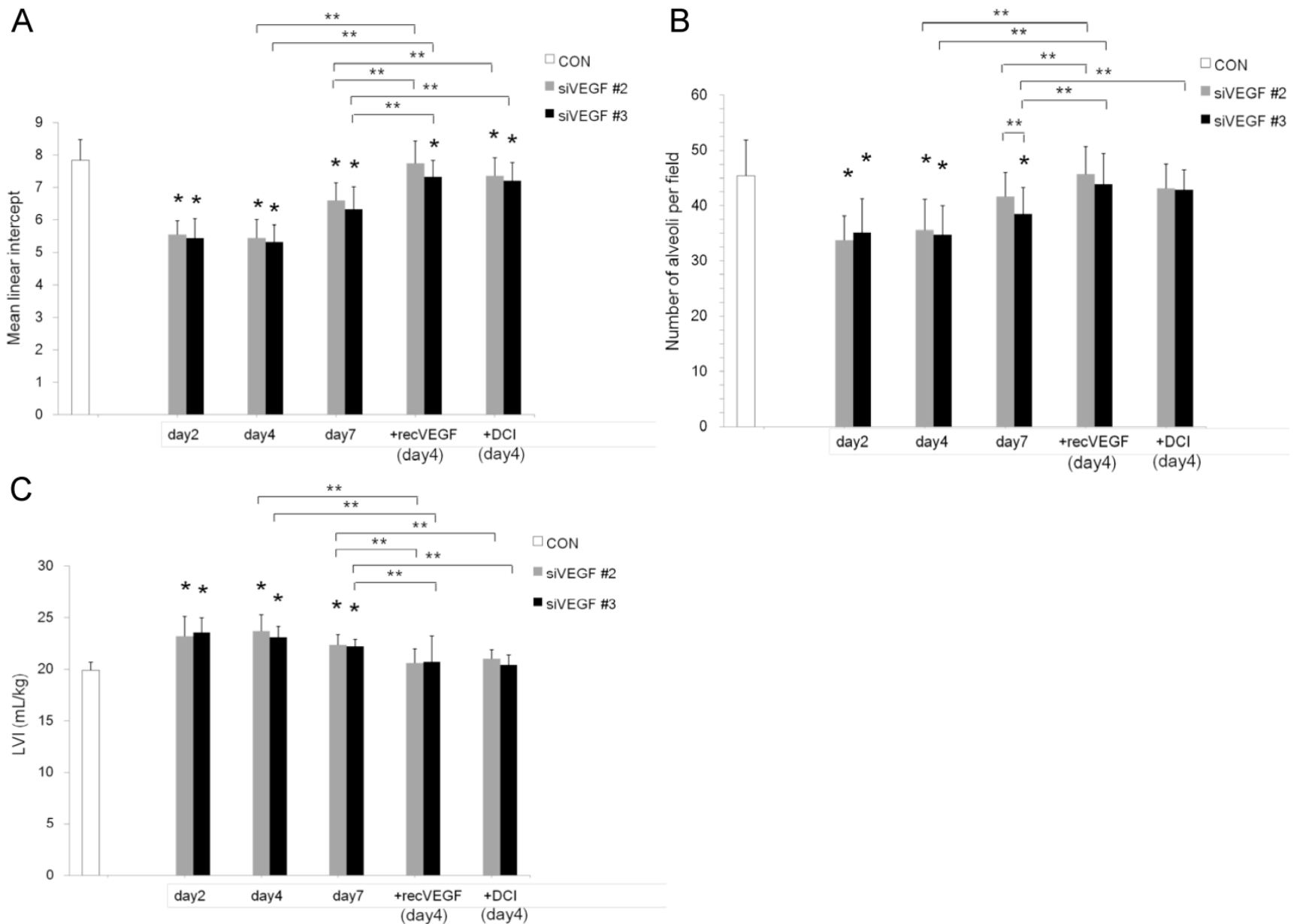

Fig 2. Air space enlargement was quantitatively analyzed. (A) Mean linear intercept was significantly decreased in comparison to the group administered a nonsilencing oligoneucleotide (CON) at days 2 and 4 after VEGF siRNAs \#2 and \#3 administration. There was a tendency towards recovery at day 7. Mean linear intercept at day 4 was significantly recovered in the groups which were administered recombinant VEGF or DCl on day 2, in comparison to the siRNAs only groups, close to the control level. $n=4$ in each group at each time point. ${ }^{*} p<0.05$ vs CON, ${ }^{* *} \mathrm{p}<0.05$ between the indicated groups. (B) Number of alveoli per field was significantly decreased in comparison to the group administered a nonsilencing oligoneucleotide (CON) at days 2 and 4 after VEGF siRNAs \#2 and \#3 administration. There was a tendency towards recovery at day 7 . Number of alveoli per field at day 4 was significantly recovered in the groups which were administered recombinant VEGF or $\mathrm{DCl}$ on day 2 , in comparison to the siRNAs only groups, close to the control level. $\mathrm{n}=4$ in each group at each time point. $* \mathrm{p}<0.05$ vs $\mathrm{CON}$, $* * \mathrm{p}<0.05$ between the indicated groups. (C) Lung volume index (LVI) was significantly increased in comparison to the group administered a nonsilencing oligoneucleotide (CON) at days 2 and 4 after VEGF siRNAs \#2 and \#3 administration. There was a tendency towards recovery at day 7 . LVI at day 4 was significantly recovered in the groups which were administered recombinant VEGF or DCl on day 2 , in comparison to the siRNAs only groups, close to the control level. $n=4$ in each group at each time point. ${ }^{*} \mathrm{p}<0.05$ vs $\mathrm{CON},{ }^{* *} \mathrm{p}<0.05$ between the indicated groups. 


\section{Western blot analysis for VEGF}

Western blot analysis showed that the level of VEGF protein expression in the lung was significantly decreased in comparison to control at days 2, 4, and 7 after VEGF siRNAs administration. The magnitude of reduction was not statistically different between the 2 sequences, siVEGF\#2 and siVEGF\#3. Administration of recombinant VEGF 2 days after VEGF siRNAs administration significantly attenuated the reduction in VEGF protein expression at day 4 in comparison to the groups which received only VEGF siRNAs (Fig. 3 A). Administration of DCI 2 days after VEGF siRNAs administration also significantly attenuated the reduction in VEGF protein expression at day 4 in comparison to the groups which received only VEGF siRNAs (Fig. 3 B). Since DCI is known to upregulate TTF-1 expression in the lung, the effect of TTF-1 siRNAs administration on VEGF expression in the lung was also examined [19]. Airway administration of TTF-1 siRNAs reduced VEGF expression in the lung at day 4 (Fig. $3 \mathrm{C}$ ), although no apparent changes in lung morphology were observed (data not shown).

\section{Immunohistochemistry and terminal deox- ynucleotidyl transferase-mediated nick end labeling (TUNEL) staining}

Analysis by immunohistochemistry showed that the proportion of alveolar septal cells with $\mathrm{Ki}-67$ positive nuclei was not significantly different in compar-

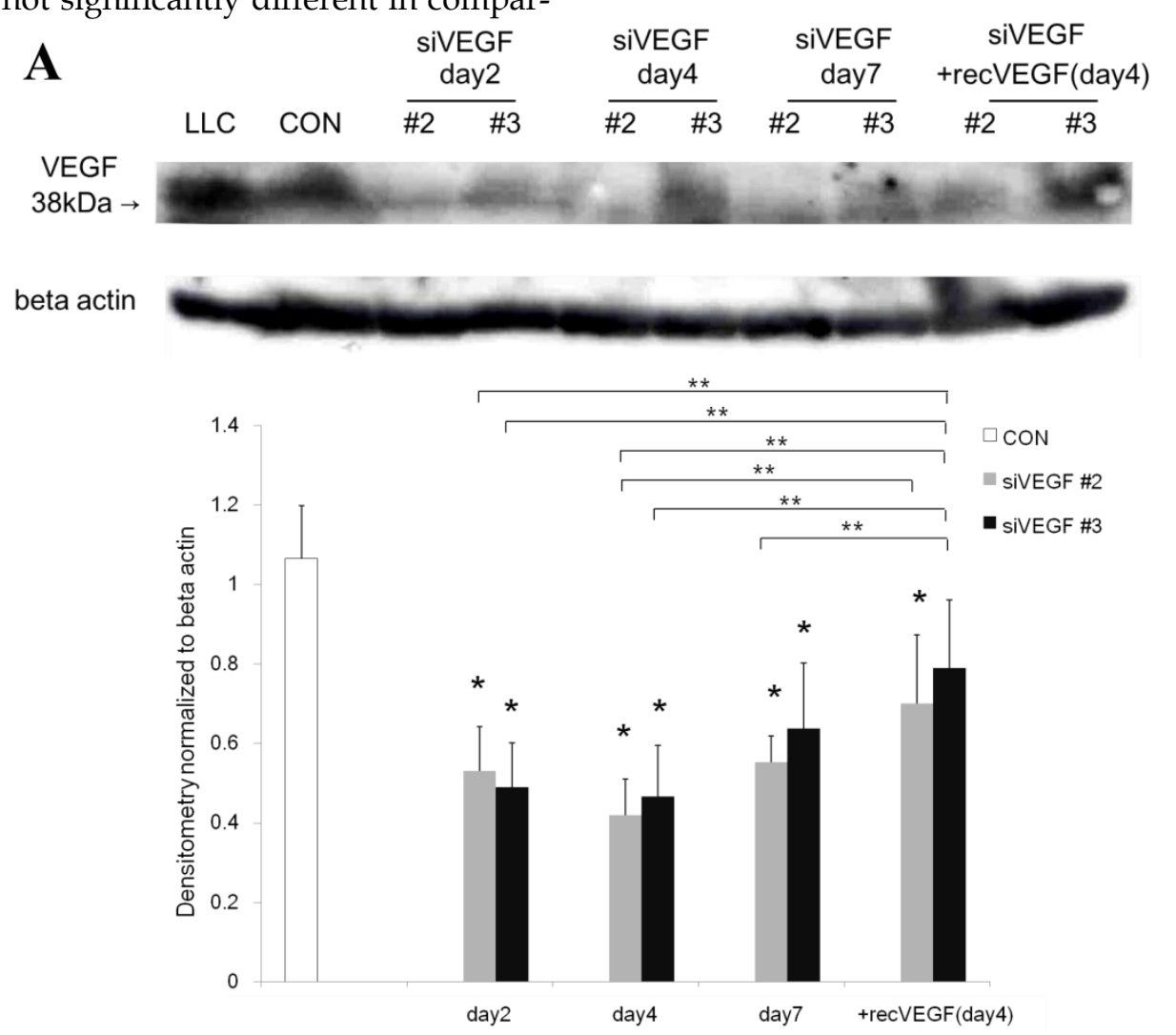

ison to control at day 4 after VEGF siRNAs administration. However, administration of recombinant VEGF or DCI, 2 days after VEGF siRNA administration, significantly increased the proportion of alveolar septal cells with Ki-67 positive nuclei at day 4 (Fig. 4A). TUNEL staining showed that the proportion of alveolar septal cells with TUNEL positive nuclei was significantly increased in comparison to control at days 2 and 4 after VEGF siRNAs administration. Administration of recombinant VEGF 2 days after VEGF siRNA administration significantly attenuated the increased in the proportion of alveolar septal cells with TUNEL positive nuclei at day 4. Administration of DCI 2 days after VEGF siRNA administration also significantly attenuated the increased the proportion of alveolar septal cells with TUNEL positive nuclei at day 4, similar to the level of control (Fig. 4B). VEGF staining showed that the proportion of VEGF positive alveolar septal cells was significantly reduced in comparison to control at days 2 and 4 after VEGF siRNAs administration. Administration of recombinant VEGF 2 days after VEGF siRNA administration significantly attenuated the reduction in the proportion of VEGF positive alveolar septal cells at day 4 . Administration of DCI 2 days after VEGF siRNA administration also significantly attenuated the reduction in the proportion of VEGF positive alveolar septal cells at day 4, similar to the level of control (Fig. 4C).

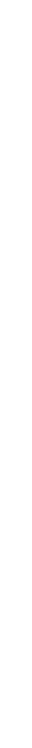



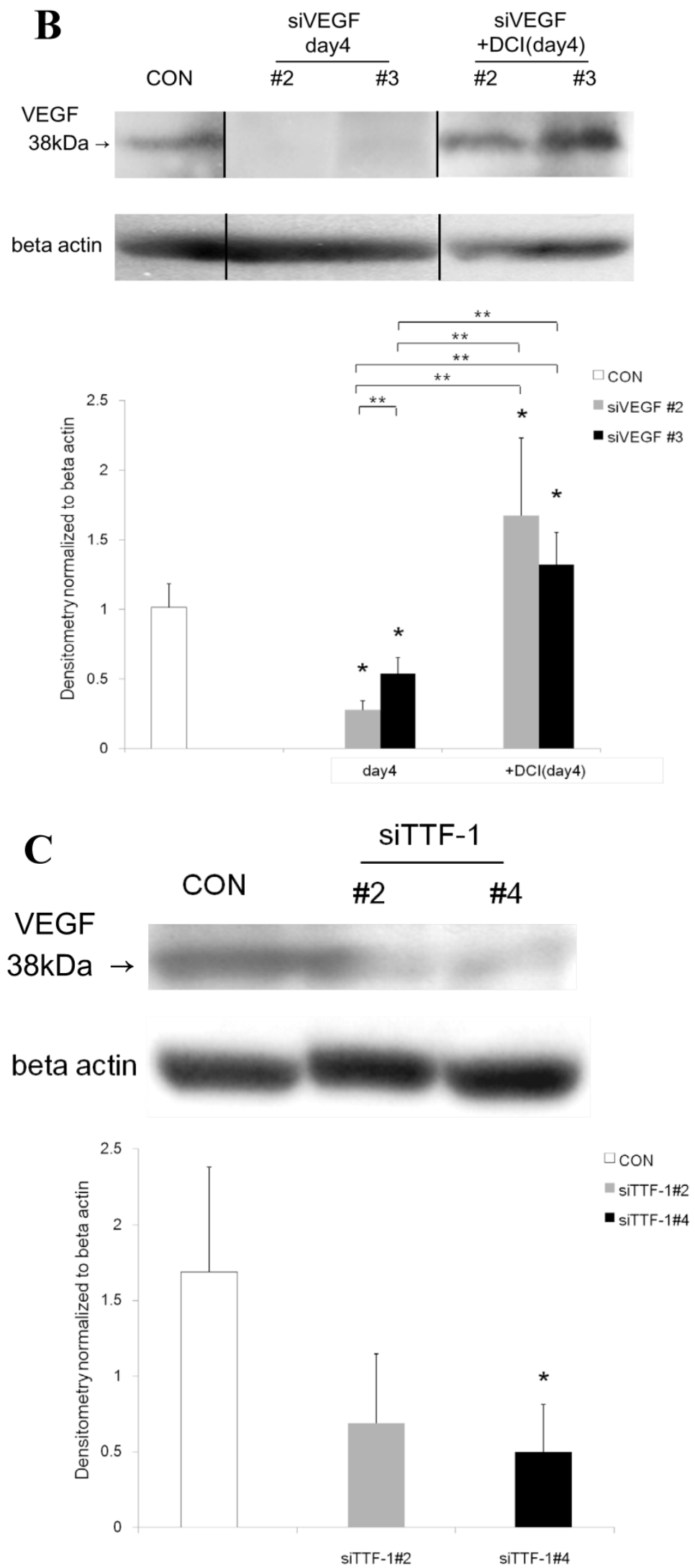

Fig 3. Expression of VEGF in lung tissue. (A) Western blot analysis showed that the level of VEGF protein was significantly decreased in comparison to the group administered a nonsilencing oligoneucleotide (CON) at days 2, 4, and 7 after VEGF siRNAs administration. Lewis lung carcinoma cell line (LLC) is also shown for comparison. Administration of recombinant VEGF 2 days after VEGF siRNA administration significantly increased the VEGF protein level at day 4, almost to the level of the CON group. (B) Administration of DCI 2 days after VEGF siRNAs administration significantly increased the VEGF protein level at day 4, almost to the level of the CON group. Lines separate different lanes from one gel. (C) Administration of TTF-I siRNAs reduced VEGF protein level 2 days after. $n=4$ in each group. ${ }^{*}<0.05$ vs CON group. 

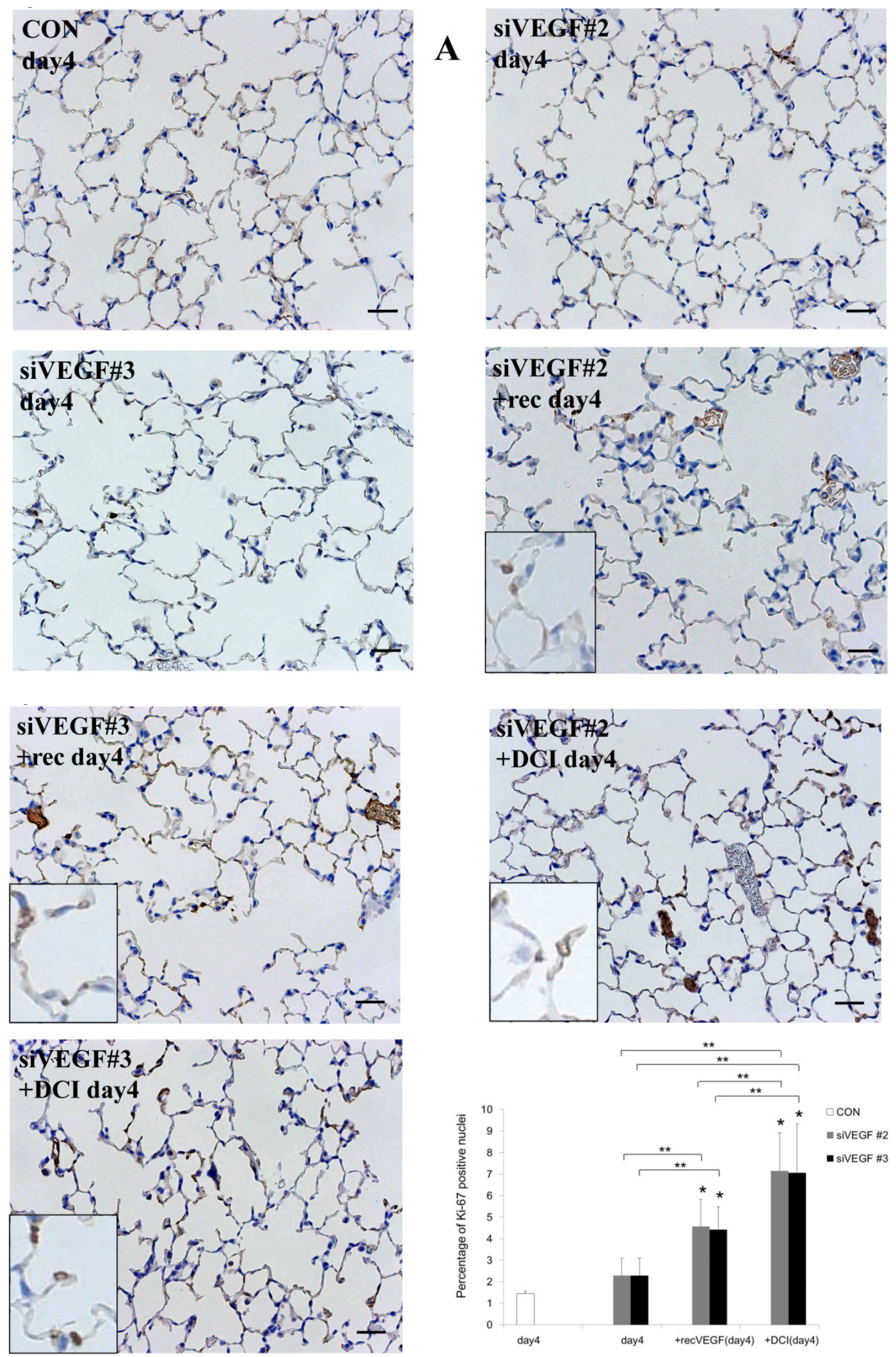

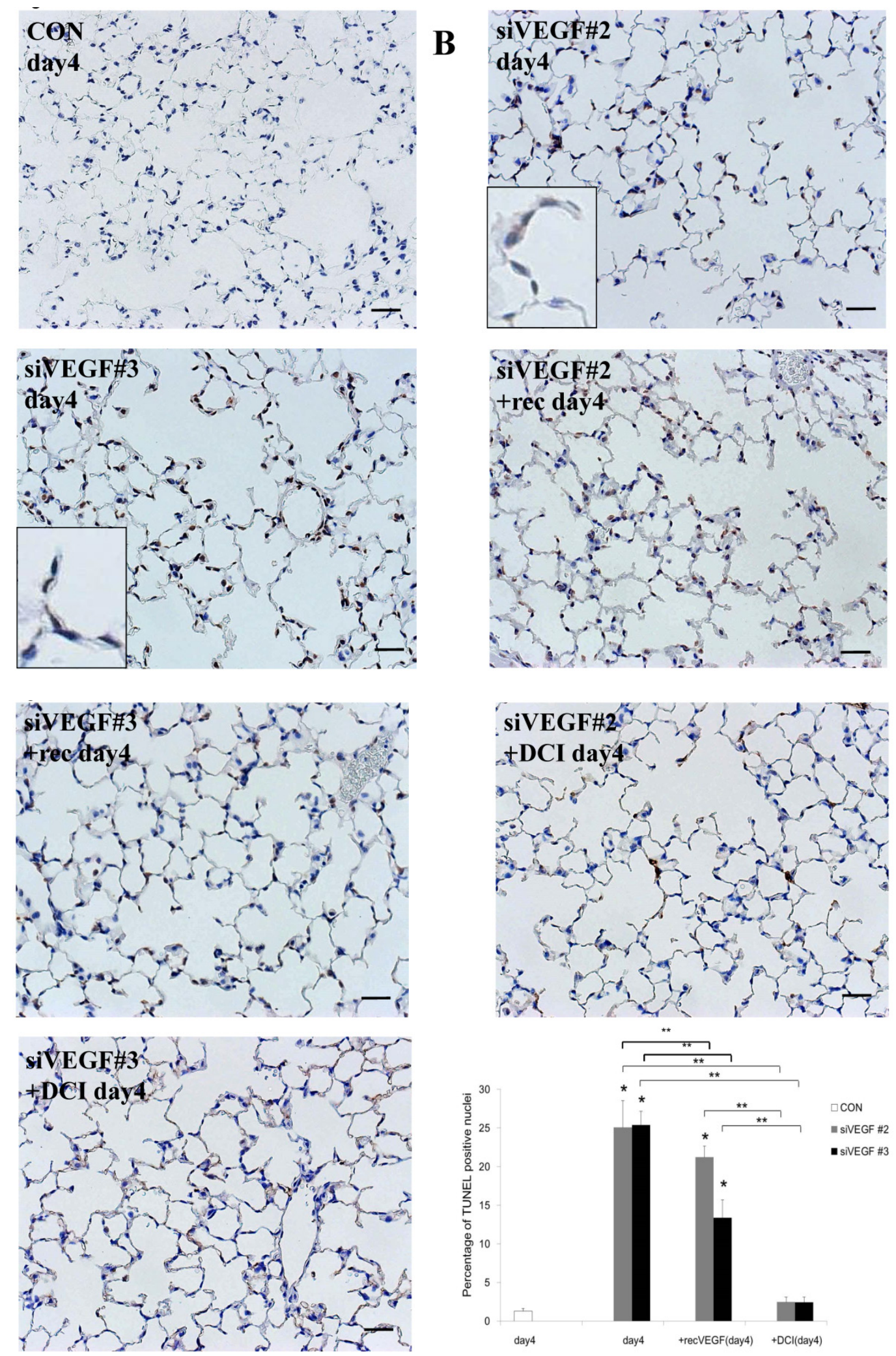

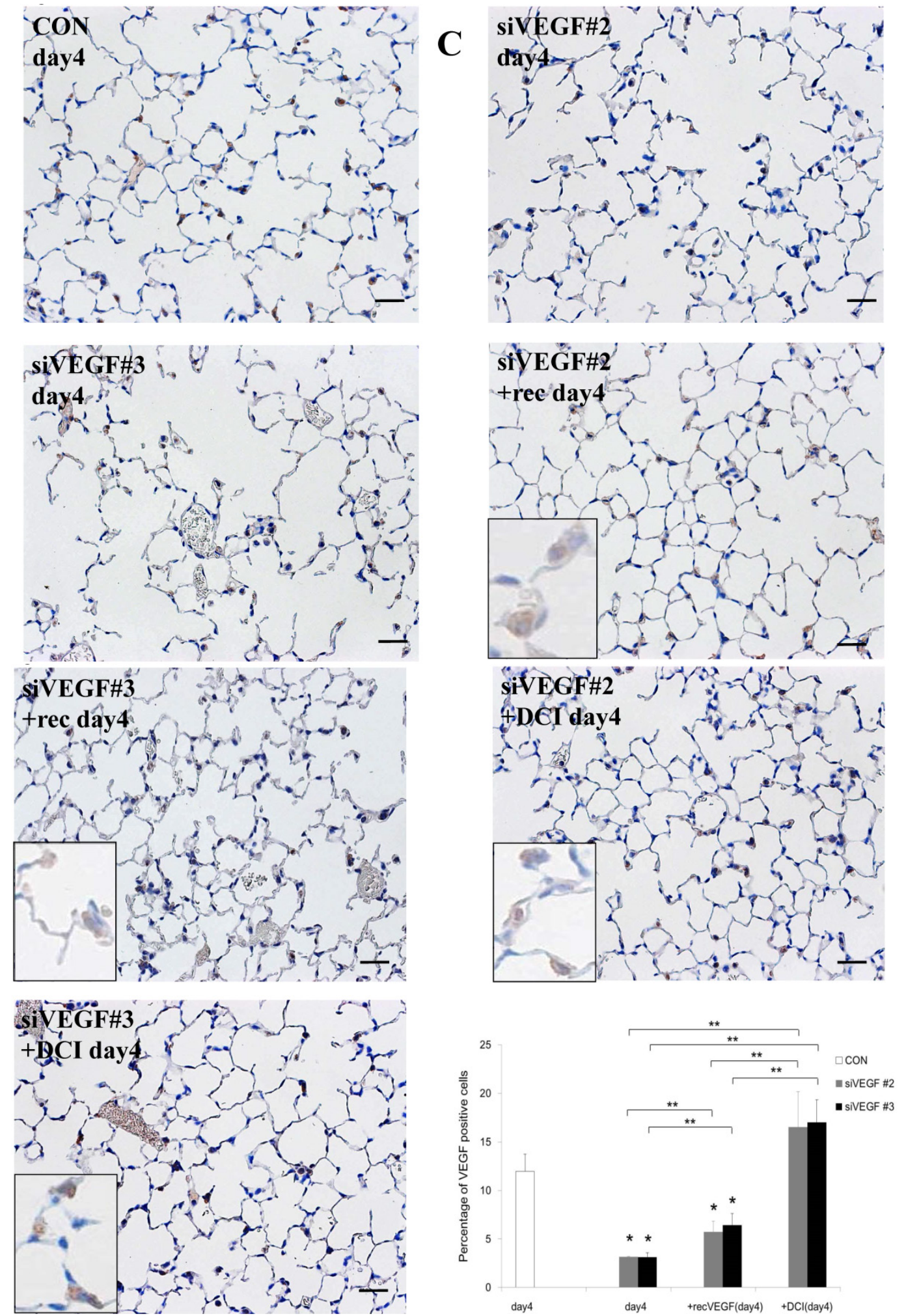

Fig 4. (A) Analysis by immunohistochemistry showed that the proportion of alveolar septal cells with Ki-67 positive nuclei was not significantly different in comparison to control at day 4 after VEGF siRNAs administration. However, administration of recombinant VEGF or DCI, 2 days after VEGF siRNA administration, significantly increased the proportion of alveolar septal cells with Ki-67 positive nuclei at day 4 (inset). (B) TUNEL staining showed that the proportion of alveolar septal cells with TUNEL positive nuclei was significantly increased in comparison to control at days 2 and 4 after VEGF siRNAs administration (inset). Administration of recombinant VEGF 2 days after VEGF siRNA administration significantly attenuated the increased the proportion of alveolar septal cells with TUNEL positive nuclei at day 4. Administration of DCl 2 days after VEGF siRNA administration also significantly attenuated the increased the proportion of alveolar septal cells with TUNEL positive nuclei at day 4, similar to the level of control. (C) VEGF staining showed that the proportion of VEGF positive alveolar septal cells was significantly reduced in comparison to control at days 2 and 4 after VEGF siRNAs administration. Administration of recombinant VEGF 2 days after VEGF siRNA administration significantly attenuated the reduction in the proportion of VEGF positive alveolar septal cells at day 4. Administration of DCI 2 days after VEGF siRNA administration also significantly attenuated the reduction in the proportion of VEGF positive alveolar septal cells at day 4, similar to the level of control (inset). Scale bars 100 microns. $\mathrm{n}=4$ in each group at each time point. $* \mathrm{p}<0.05$ versus $\operatorname{CON}$ (control group), ${ }^{* *} \mathrm{p}<0.05$ between the indicated groups. 


\section{Comment}

VEGF is a critical angiogenic factor in maintaining normal tissue vasculature, endothelial cell growth, and survival [20]. Cigarette smoke exposure has been associated with reduced VEGF and VEGF receptor 2 expression in severe emphysema patients, and in rodent models [21, 22]. Moreover, reduced VEGF has been implicated in the destruction of alveolar wall components including the microvasculature $[4,21$, 23-25].

In the lung, VEGF is synthesized by the alveolar epithelial cells, bronchial epithelial cells and alveolar macrophages, and is reported to have versatile effects on the various cells types which constitute the lung [26]. The effects on the individual cell types, direct and indirect, are still to be defined [27]. Since the lung is exposed constantly to external stresses, VEGF may have versatile direct and indirect biological functions in the maintenance of lung repair and homeostasis.

In the present study, airway administration of VEGF siRNAs transiently reduced VEGF protein expression in the lung and induced transient air space enlargement. Immunohistochemistry and TUNEL staining studies suggested that the reduction in VEGF levels were predominantly in the alveolar septal cells, and that the air space enlargement was primarily due to increased apoptosis of alveolar septal cells rather than reduction in the baseline rate of proliferation. There are multiple models of emphysema, namely lung air space enlargement, but the pathogenesis of clinically encountered emphysema may be different or more multifaceted. In the present study, the kinetics of air space enlargement was much faster in comparison to the previously reported models of pharmacological VEGF pathway blockade. The fact that the response to VEGF siRNA administration was fast implies that the turnover rate of cells in the lung is high. Airspace enlargement induced by VEGF receptor blockade takes longer to develop, suggesting the possible differences in the route of administration, and/or suppression of VEGF expression rather than the blockade of its receptors. Furthermore, the uniformity of air space enlargement likely depends on the distribution of administered siRNAs and may be improved by further optimization of methods of application. Nevertheless, the model in the present study may be useful in dissecting the involvement of VEGF in pulmonary emphysema. Further studies including the evaluation of repetitive administrations of VEGF siRNAs need to be done as well as the dissection of potential involvement of different VEGF isoforms. Also, in order to appropriately address the structural remodeling of the alveolar septa during air space enlargement and the subsequent recovery, we assume that more detailed time course studies are necessary, particularly with inclusion of earlier time points, as well as later time points to see if the air space enlargement eventually reverts to normal. Although morphologically we did not observe any signs of inflammation such as the presence of infiltrating cells after administration of VEGF siRNAs, the involvement and/or modification of pathways associated with inflammation are also needed.

In terms of potential therapeutics for pulmonary emphysema, all-trans retinoic acid has been reported to reverse emphysema in rats [28] while contradictory results have also been reported [2, 29, 30]. Keratinocyte growth factor has been shown to reverses elastase induced emphysema at least in part through induction of VEGF [31].

In the present study, sequential airway administration of recombinant VEGF protein or DCI attenuated the airspace enlargement induced by VEGF siRNAs as well as the reduction in VEGF expression. Immunohistochemistry studies suggested that airway administration of both recombinant VEGF protein and DCI increased proliferation of alveolar septal cells, and attenuated the apoptosis induced by VEGF siRNAs, at least in part by restoration of baseline VEGF expression. Recombinant VEGF or DCI was administered 2 days after the administration of siRNA at which point air space enlargement had already occurred. This indicates that these agents were able to reverse rather than prevent the air space enlargement induced by VEGF depletion.

Airway administration of VEGF has been shown to improve lung maturation in mouse fetus without inducing angiogenesis or vascular leakage in the alveolar septa [15]. It seems that the applied VEGF remains restricted to the alveolar compartment with very little spill-over into the interstitium or the circulation [32]. The effect of externally applied VEGF per se is expected to be brief. Hence we speculate that application of VEGF induced a chain of signaling events leading to the recovery of VEGF production by the lung parenchymal cells. This result suggests that VEGF application may attenuate air space enlargement in pulmonary emphysema. However, clinical emphysema consists of a more permanent enlargement of airspaces that would not be expected to reverse so quickly. The transient nature of the siRNAs is likely one reason why the lung showed a tendency towards prompt restoration. Repetitive administration studies may address some of these issues. Furthermore, VEGF may also induce lung injury depending on the context [33]. The effect of long-term repetitive applications needs to be studied since persistence of excess VEGF in the lung could cause alterations in vascular permeability and in lung remodel- 
ing [34].

The mechanisms of VEGF induction in the lung by DCI requires further investigation. VEGF mRNA expression has been shown to be reduced in TTF-1 phosphorylation mutant mice [35]. In the present study, we found that TTF-1 siRNA administration reduced VEGF expression level in the lung. Since DCI is known to induce TTF-1 in vitro [12], induction of VEGF by DCI may in part be mediated through TTF-1. However, air space enlargement was not apparent on histology after TTF-1 siRNAs administration, and therefore, other pathways, likely downstream of VEGF needs to be further examined.

\section{Acknowledgements}

The authors thank Kei Tsujioka, Division of General Thoracic Surgery, School of Medicine, Keio University, for her expertise in animal experiments. This work was supported in part by grant in aid from the Ministry of Education, Culture, Sports, Science, and Technology-Japan, and the School of Medicine, Keio University fund for the promotion of science.

\section{Competing Interests}

The authors have declared that no competing interest exists.

\section{References}

1. Taraseviciene-Stewart L, Voelkel NF. Molecular pathogenesis of emphysema. J Clin Invest. 2008; 118: 394-402.

2. Monacci WT, Merrill MJ, Oldfield EH. Expression of vascular permeability factor/vascular endothelial growth factor in normal rat tissue. Am J Physiol. 1993; 264: C995-1002.

3. Voelkel NF, Vandivier RW, Tuder RM. Vascular endothelial growth factor in the lung. Am J Physiol Lung Cell Mol Physiol. 2006; 290: L209-221.

4. Kasahara Y, Tuder RM, Cool CD, et al. Endothelial cell death and decreased expression of vascular endothelial growth factor and vascular endothelial growth factor receptor 2 in emphysema. Am J Respir Crit Care Med. 2001; 163: 737-744

5. Koyama S, Sato E, Haniuda M, et al. Decreased level of vascular endothelial growth factor in bronchoalveolar lavage fluid of normal smokers and patients with pulmonary fibrosis. Am J Respir Crit Care Med. 2002; 166: 382-385.

6. Petrache I, Natarajan V, Zhen L, et al. Ceramide upregulation causes pulmonary cell apoptosis and emphysema-like disease in mice. Nat Med. 2005; 11: 471-472.

7. Kasahara Y, Tuder RM, Taraseviciene-Stewart L, et al. Inhibition of VEGF receptors causes lung cell apoptosis and emphysema. J Clin Invest. 2000; 106: 1309-1310.

8. Petrache I, Fijalkowska I, Zhen L, et al. A novel antiapoptotic role for alpha1-antitrypsin in the prevention of pulmonary emphysema. Am J Respir Crit Care Med. 2006; 173: 1222-1228.

9. Tuder RM, Zhen L, Cho CY, et al. Oxidative stress and apoptosis interact and cause emphysema due to vascular endothelial growth factor receptor blockade. Am J Respir Cell Mol Biol. 2003; 29: 88-97.

10. Tang K, Rossiter HB, Wagner PD, et al. Lung-targeted VEGF inactivation leads to an emphysema phenotype in mice. J Appl Physiol. 2004; 97: 1559-1566.

11. Kolla V, Gonzales LW, Gonzales J, et al. Thyroid transcription factor in differentiating type II cells: regulation, isoforms, and target genes. Am J Respir Cell Mol Biol. 2006; 36: 213-225.

12. Takahashi $Y$, Izumi $Y$, Kohno $M$, et al. Thyroid transcription factor-1 influences the early phase of compensatory lung growth in adult mice. Am J Respir Crit Care Med. 2010:181:1397-406

13. Massaro D, Massaro GD, Clerch LB. Noninvasive delivery of small inhibitory RNA and other reagents to pulmonary alveoli in mice. Am J Physiol Lung Cell Mol Physiol. 2004; 287: L1066-1070.

14. Zhang X, Turnell AS, Gorbea C, et al. The targeting of the proteasomal regulatory subunit S2 by adenovirus E1A causes inhibition of proteasomal activity and increased p53 expression. J Biol Chem. 2004; 279: 25122-25133.
15. Compernolle V, Brusselmans K, Acker T, et al. Loss of HIF-2alpha inhibition of VEGF impair fetal lung maturation, whereas treatment with VEGF prevents fatal respiratory distress in premature mice. Nat Med. 2002; 8: 1329.

16. Scherle W. A simple method for volumetry of organs in quantitative stereology. Mikroskopie. 1970; 26: 57-60.

17. Thurlbeck WM, Angus GE. The variation of Reid index measurements within the major brochial tree. Am Rev Respir Dis. 1967; 95: 551-555.

18. Inselman LS, Padilla-Burgos LB, Teichberg S, Spencer H. Alveolar enlargement in obesity-induced hyperplastic lung growth. J Appl Physiol. 1998; 65: 2291-2296.

19. Takahashi Y, Izumi Y, Kohno M, et al. Airway administration of dexamethasone, 3'-5'-cyclic adenosine monophosphate, and isobutylmethylxanthine facilitates compensatory lung growth in adult mice. Am J Physiol Lung Cell Mol Physiol 2011;300:L453-61.

20. Gerber HP, McMurtrey A, Kowalski J, et al. Vascular endothelial growth factor regulates endothelial cell survival through the phosphatidylinositol 3'-kinase/Akt signal transduction pathway. Requirement for Flk-1/KDR activation. J Biol Chem. 1998; 273: 30336-30343.

21. Marwick JA, Stevenson CS, Giddings J, et al. Cigarette smoke disrupts VEGF165-VEGFR-2 receptor signaling complex in rat lungs and patients with COPD: morphological impact of VEGFR-2 inhibition. Am J Physiol Lung Cell Mol Physiol. 2006; 290: L897-908.

22. Suzuki M, Betsuyaku T, Nagai K, et al. Decreased airway expression of vascular endothelial growth facor in cigarette smoke-induced emphysema in mice and COPD patients. Inhal Toxicol. 2008; 20: 349-359.

23. Spira A, Beane J, Pinto-Plata V, et al. Gene expression profiling of human lung tissue from smokers with severe emphysema. Am J Respir Cell Mol Biol. 2004; 31: 601-610.

24. Kanazawa,H, Yoshikawa J. Elevated oxidative stress and reciprocal reduction of vascular endothelial growth factor levels with severity of COPD. Chest. 2005; 128: 3191-3197.

25. Ho YS, Chen $\mathrm{CH}$, Wang $\mathrm{YJ}$, et al. Tobacco-specific carcinogen 4-(methylnitrosamino)-1-(3-pyridyl)-1-butanone (NNK) induces cell proliferation in normal human bronchial epithelial cells through NFkappaB activation and cyclin D1 up-regulation. Toxicol. Appl Pharmacol. 2005; 205: 133-148.

26. Brown KR, England KM, Goss KL, et al. VEGF induces airway epithelial cell proliferation in human fetal lung in vitro. Am J Physiol Lung Cell Mol Physiol. 2001; 281: L1001-1010.

27. Raoul W, Chailley-Heu B, Barlier-Mur AM, et al. Effects of vascular endothelial growth factor on isolated fetal alveolar type II cells. Am J Physiol Lung Cell Mol Physiol. 2004; 286: L1293-1301.

28. Massaro GD, Massaro D. Retinoic acid treatment abrogates elastase-induced pulmonary emphysema in rats. Nat Med. 1997; 3: 675-677.

29. March TH, Cossey PY, Esparza DC, et al. Inhalation administration of all-trans-retinoic acid for treatment of elastase-induced pulmonary emphysema in Fischer 344 rats. Exp Lung Res. 2004; 30: 383-404.

30. Fujita $M, Y e Q$, Ouchi $H$, et al. Retinoic acid fails to reverse emphysema in adult mouse models. Thorax. 2004; 59: 224-230.

31. Yildirim AO, Muyal V, John G, et al. Palifermin induces alveolar maintenance programs in emphysematous mice. Am J Respir Crit Care Med. 2010; 181: 705-717.

32. Kaner RJ. Crystal RG. Compartmentalization of vascular endothelial growth factor to the epithelial surface of the human lung. Mol Med. 2001; 7: 240-246.

33. Tuder RM, Yun JH. Vascular endothelial growth factor of the lung: friend or foe. Curr Opin Pharmacol. 2008; 8: 255-260.

34. Le Cras TD, Spitzmiller RE, Albertine KH, et al. VEGF causes pulmonary hemorrhage, hemosiderosis, and air space enlargement in neonatal mice. Am J Physiol Lung Cell Mol Physiol. 2004; 287: L134-142.

35. De Felice M, Esposito L, Pucci B, et al. Biochemical characterization of a CDC6-like protein from the crenarchaeon Sulfolobus solfataricus. J Biol Chem. 2003; 278: 46424-46431. 